\title{
Hot \& cold dust in M31: the resolved SED of Andromeda
}

\author{
Brent Groves $^{1}$, Oliver Krause ${ }^{1}$ and the MPIA Herschel Andromeda \\ Team \\ ${ }^{1}$ Max Planck Institute for Astronomy, Königstuhl 17, D-69117 Heidelberg, Germany
}

email: brent@mpia.de, krause@mpia.de

\begin{abstract}
Due to its proximity, the Andromeda galaxy (M31, NGC 224) offers a unique insight into how the spectra of stars, dust, and gas combine to form the integrated Spectral Energy Distribution (SED) of galaxies. We introduce here Herschel Space Observatory PACS and SPIRE photometric observations of M31 which cover the far-infrared to sub-mm wavelengths $(70-500 \mu \mathrm{m})$. These new observations reveal that the total IR luminosity of M31 is relatively weak, with $L_{\mathrm{IR}}=10^{9.65} L_{\odot}$, only $10 \%$ of the total luminosity of M31. However, as seen in the previous studies of M31, the IR luminosity is dominated by a $10 \mathrm{kpc}$ ring in all Herschel bands. This is distinct from the optical, where the bulge in the central $2 \mathrm{kpc}$, dominates the luminosity, clearly demonstrating how different components at distinct positions in a galaxy contribute to make the integrated SED.
\end{abstract}

Keywords. Galaxies:individual:M31,ISM:dust

\section{Introduction}

The Andromeda galaxy (M31) has helped forge our understanding of the association of dust with gas and stars on galactic scales. The seminal work of Baade \& Gaposchkin (1963) noted that in Andromeda the dust (as seen through extinction) was predominantly associated with young stars and hence star formation. Yet it was with far-infrared space telescopes that the dust revealed itself. The IRAS observations of M31 (Habing et al. 1984, Soifer et al. 1986, Walterbos \& Schwering 1987, Xu \& Helou 1996a,b) found the IR luminosity of M31 was relatively weak compared to many other galaxies found in the IRAS surveys. The IRAS survey found the IR maps dominated by the $10 \mathrm{kpc}$ ring, as also found at $175 \mu \mathrm{m}$ in the ISO survey of M31 (Haas et al. 1998).

The Spitzer Space Telescope view of M31 took this IR image to the next level of resolution, with the $10 \mathrm{kpc}$ ring clearly visible in the IRAC $8 \mu \mathrm{m}$ band (Barmby et al. 2006), and in the longer wavelength MIPS bands (Gordon et al. 2006). In addition to the larger $10 \mathrm{kpc}$ ring, the MIPS imagery also revealed the central peak, and inner spirals and outer loops. Using this Spitzer data, in association with GALEX and SDSS imaging, Montalto et al. (2009) modelled the dust of M31, determining the dust mass distribution and importantly the heating of the dust across the disk. They found that over the majority of the disk the dust was heated predominantly by optical photons and stars older than 1 Gyr, as suggested in the earlier work by Xu \& Helou (1996b).

In this paper we continue this investigation into the far-infrared properties of M31 with Herschel Space Observatory (Pilbratt et al. 2010) observations of the Andromeda galaxy. This data has given us an unprecedented look into the long wavelength emission from M31 and demonstrates that, overall, M31 has a low total IR luminosity and relatively low total dust mass. However, even with cold dust (long wavelengths), the $10 \mathrm{kpc}$ ring 


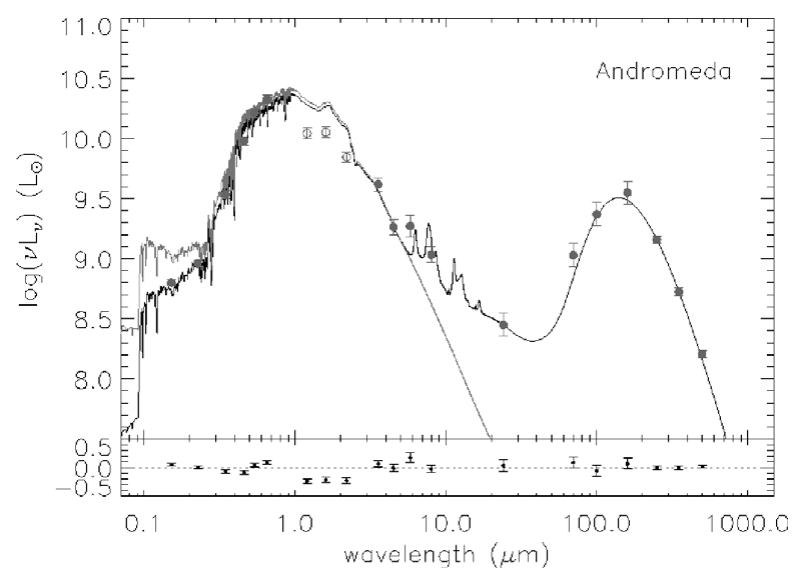

Figure 1. The spectral energy distribution of Andromeda (filled points), and the best fit model (black curve) from the SED fitting code MAGPHYS (da Cunha et al. 2008). Note that the 2MASS data (empty circles) were not used in determining the best fit due to the smaller aperture used. The solid curve shows the best model fit, while the grey line shows the unattenuated stellar spectrum. In the lower plot we show the offset of the data from the best fit model.

dominates the emission, but dust emission can be found out to radii even further than this. For full details of this work, we refer the reader to Groves et al. (in prep.).

\section{The Integrated SED of Andromeda}

The Andromeda galaxy was observed in all 6 Herschel photometric bands (PACS 70, 100, and $160 \mu \mathrm{m}$ and SPIRE 250, 350, and $500 \mu \mathrm{m}$ as a Guaranteed Time (GT1) program. $\mathrm{A} \sim 3^{\circ} \times 1^{\circ}$ region centred on M31 was observed in slow parallel mode $\left(20^{\prime \prime} / \mathrm{s}\right)$ for a total time of $\sim 24$ hours (86412 seconds). The full observations and reduction are described in detail in Krause et al. (in prep.).

Using literature data (UV from Gil de Paz et al. (2007), optical from Walterbos \& Kennicutt (1987), near-IR from Jarrett et al. (2003), and the mid-IR from Barmby et al. (2006) and Gordon et al. (2006)) and the new Herschel data, it is now possible to obtain the full UV-IR integrated spectral energy distribution (SED) of the Andromeda galaxy, enabling a comparison of our nearest massive neighbour with more distant galaxies. We obtained the full SED of M31 within a $r_{\mathrm{maj}} \sim 95^{\prime}$ ellipse, with an inclination of $75^{\circ}$ and a P.A. of $37.7^{\circ}$, shown by the filled points in Figure 1 .

Figure 1 also shows the best fit model SED from the SED fitting code MAGPHYS (da Cunha et al. 2008). Due to the significantly smaller aperture used to determine the 2MASS fluxes and the observable offset between these fluxes and the $\mathrm{R}$ and $3.6 \mu \mathrm{m}$ bands, we have not included these in the determination of the best fit model. The offset between the best fit model and the observations can be seen in the lower part of Figure 1 , including the offset of the 2MASS points.

It is immediately clear from the integrated SED that M31 is an early type spiral, with a relatively low UV and IR luminosity (c.f. the work of Mutch et al. 2011, who compare Andromeda with SDSS galaxies, and found that it fell in the "green valley"). The total luminosity of M31 from the best-fit model is $\log \left(\mathrm{L}_{*} / \mathrm{L}_{\odot}\right) \sim 10.61$. Only $\sim 10 \%$ of the radiation in M31 has been processed by dust, with $\mathrm{L}_{\text {dust }}=4.44_{-0.10}^{+0.15} \times 10^{9}\left(\mathrm{~L}_{\odot}\right)$, and the subsequent radiation from the dust is predominantly cool, peaking at $\sim 160 \mu \mathrm{m}$. The total mass of dust returned by the model is $\log M_{\mathrm{d}}=7.43 \pm 0.06\left(M_{\odot}\right)$, supporting the idea that M31 is a dust poor galaxy. The mean cold dust temperature in M31 is $16.8_{-0.7}^{+0.5} \mathrm{~K}$, 


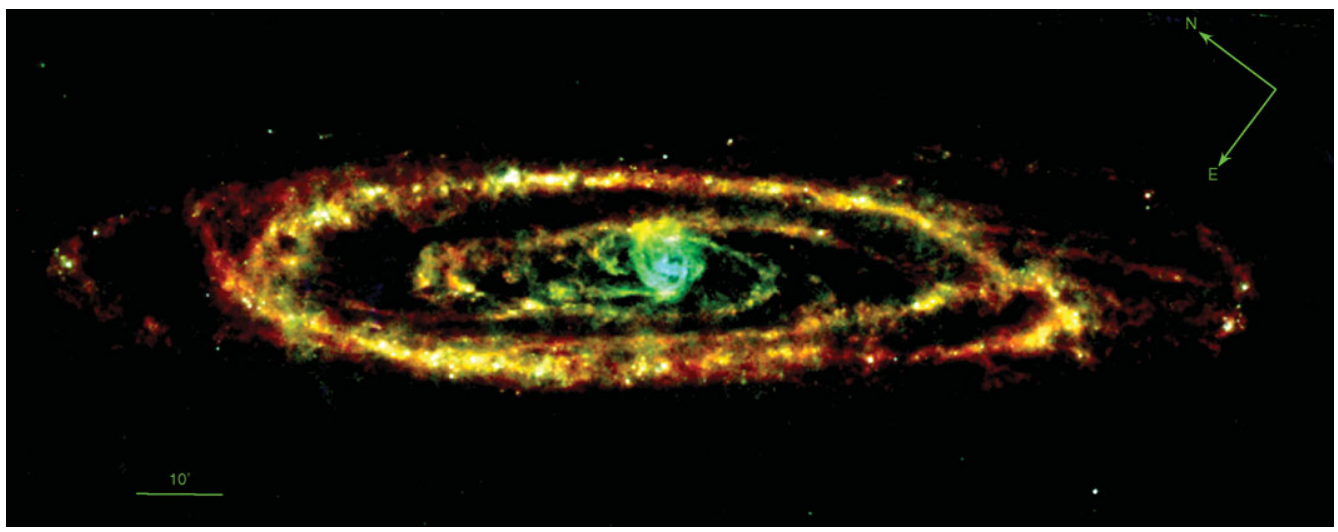

Figure 2. Far-infrared image of Andromeda using the Herschel bands showing PACS $70 \mu \mathrm{m}$, PACS $100 \mu \mathrm{m}$, and SPIRE $250 \mu \mathrm{m}$, all convolved to SPIRE $250 \mu \mathrm{m}$ resolution. All three bands have the same square root scaling from $10 \mathrm{MJy} / \mathrm{sr}$ to $150 \mathrm{MJy} / \mathrm{sr}$ The angular scale is shown by the 10 ' bar in the lower right.

as expected with the peak of the IR bump at such long wavelengths. Altogether, M31 is a relatively dust-poor spiral with relatively cool dust, mostly because it is dominated by the emission from the diffuse ISM.

\section{The resolved SED of Andromeda}

The unprecedented spatial resolution of Herschel $\left(6-8^{\prime \prime}\right.$, or $20-60 \mathrm{pc}$ at $\left.70-250 \mu \mathrm{m}\right)$, enables us to go further and determine the heating of the dust at the scales of HiI regions and giant molecular clouds, and obtain the SED of Andromeda on small scales. In Figure 2 we show a three-colour far-infrared image of M31, using the PACS 70 and $100 \mu \mathrm{m}$ and SPIRE $250 \mu \mathrm{m}$ bands. All three bands have the same square root scaling from $15 \mathrm{MJy} /$ to $150 \mathrm{MJy} / \mathrm{sr}$.

Clearly visible in the image is the $10 \mathrm{kpc}$ starforming ring of M31 and its spiral structure, with bright white points indicating positions of HII regions. Outside the $10 \mathrm{kpc}$ ring, little dust emission is seen at these levels of surface brightness, except for the extended loops along the major axis. Inside the ring, the inner spiral arms are visible, linking the ring to both the nuclear and bulge emission at the centre. These features have been visible in both the earlier IRAS and Spitzer MIPS far-IR maps of M31, yet never before at such spatial resolution at these wavelengths. This image clearly demonstrates which regions contribute at which wavelengths, with the $10 \mathrm{kpc}$ ring dominating long wavelengths but the central region dominated by the shortest wavelengths.

This is more clear when we fit a simple modified blackbody, $F_{\nu} \propto B_{\nu}\left(\mathrm{T}_{\mathrm{d}}\right)\left(\frac{\nu}{\nu_{0}}\right)^{\beta}$, to the 100-500 $\mu \mathrm{m}$ data. By minimizing over the other parameters, we determined the dust temperature $T_{\mathrm{d}}$ as a function of radius, shown in Figure 3. The radial variation quantitatively displays what was clear in Figure 2, with a high temperature in the central $2 \mathrm{kpc}$ where the bulge of M31 is, while the disk of M31 has an approximately constant temperature $\left(T_{\mathrm{d}} \approx 17 \pm 1 \mathrm{~K}\right)$. This even includes the $10 \mathrm{kpc}$ ring, where little variation is seen with the mean $T_{\mathrm{d}}$, though clearly there are individual hot HII regions within the ring. To the right of the radial temperature we show the integrated IR SEDs of the central $2 \mathrm{kpc}$ (the bulge), and between 9 and $11 \mathrm{kpc}$ (the ring) and the best fitting simple modified blackbody. While these two SEDs demonstrate the difference in dust temperatures between the two regions, they also reveal the relative contribution of these 

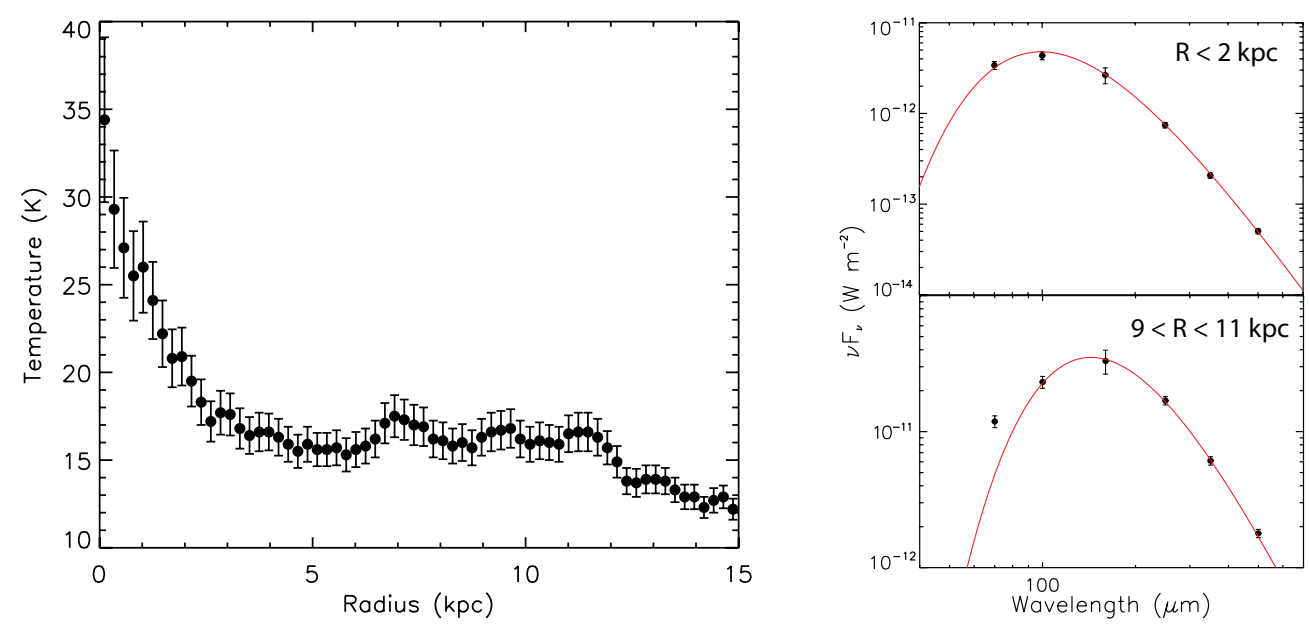

Figure 3. Left: Radial variation of mean temperature in M31. The temperature is determined by fitting the median flux in all bands in radial bins of $1^{\prime}(0.23 \mathrm{kpc})$ width using elliptical annuli. The error bars show the 16-84 percentiles of marginalized PDFs. Right: The Herschel IR SED from the central $2 \mathrm{kpc}(t o p)$ and from the $10 \mathrm{kpc}$ ring (bottom) as labelled.

regions to each band, with the overall bulge IR emission an order of magnitude weaker than the ring, but having a relatively greater (though still weak) contribution at the shortest wavelengths.

As a final note, we point out that this work presents only a small fraction of the available information with these new Herschel images. With existing optical, GALEX, Spitzer, HI, radio, and CO data, and the currently ongoing Pan-chromatic Hubble Andromeda Treasury survey which will cover roughly a third of the star forming disk, using HST 6 filters from the UV-NIR, in addition to the Herschel images presented here, there exists a seemingly-overwhelming wealth of data on the Andromeda galaxy that should prove to be a heritage in understanding the connections between stars, gas, and dust and how they combine to make a galaxy.

\section{References}

Baade, W. \& Gaposchkin, C. H. P. 1963, Evolution of stars and galaxies., by Baade, W; Gaposchkin, C. Cambridge, Harvard University Press

Barmby, P. et al. 2006, ApJL, 650, L45

da Cunha, E., Charlot, S., \& Elbaz, D. 2008, MNRAS, 388, 1595

Gil de Paz, A., Boissier, S., Madore, B. F., et al. 2007, ApJS, 173, 185

Gordon, K. D., et al. 2006, ApJL, 638, L87

Haas, M., Lemke, D., Stickel, M., et al. 1998, A\& A, 338, L33

Habing, H. J., Miley, G., Young, E., et al. 1984, ApJL, 278, L59

Jarrett, T. H., Chester, T., Cutri, R., Schneider, S. E., \& Huchra, J. P. 2003, AJ, 125, 525

Montalto, M., Seitz, S., Riffeser, A., et al. 2009, A\&A A, 507, 283

Mutch, S. J., Croton, D. J., \& Poole, G. B. 2011, ApJ, 736, 84

Pilbratt, G. L., Riedinger, J. R., Passvogel, T., et al. 2010, A\&A, 518, L1

Soifer, B. T., Rice, W. L., Mould, J. R., et al. 1986, ApJ, 304, 651

Xu, C. \& Helou, G. 1996a, ApJ, 456, 152

Xu, C. \& Helou, G. 1996b, ApJ, 456, 163

Walterbos, R. A. M. \& Kennicutt, R. C., Jr. 1987, A\& $A S, 69,311$

Walterbos, R. A. M. \& Schwering, P. B. W. 1987, A\&ऽA, 180, 27 


\section{Discussion}

Chakrabarti: Do you consider a range of temperatures? The reason I'm asking is that Chris McKee and I found that if you estimate masses assuming a single temperature, you would get a different answer than if you considered a self-consistent distribution of temperatures.

Groves: I agree. The simple models would not be able to give accurate masses. However, you can use simple modified blackbodies as a physically-motivated parameterization of the far IR colours. They are useful so long as you don't over-interpret the results.

WANG: Since M31 is highly inclined, do you see any evidence for projected dust features from the front side of galactic disk.

Groves: While most of the disk is optically thin we can see on the 3-colour Herschel image of M31 a portion where we have the disk dust emission projected onto the bulge. 